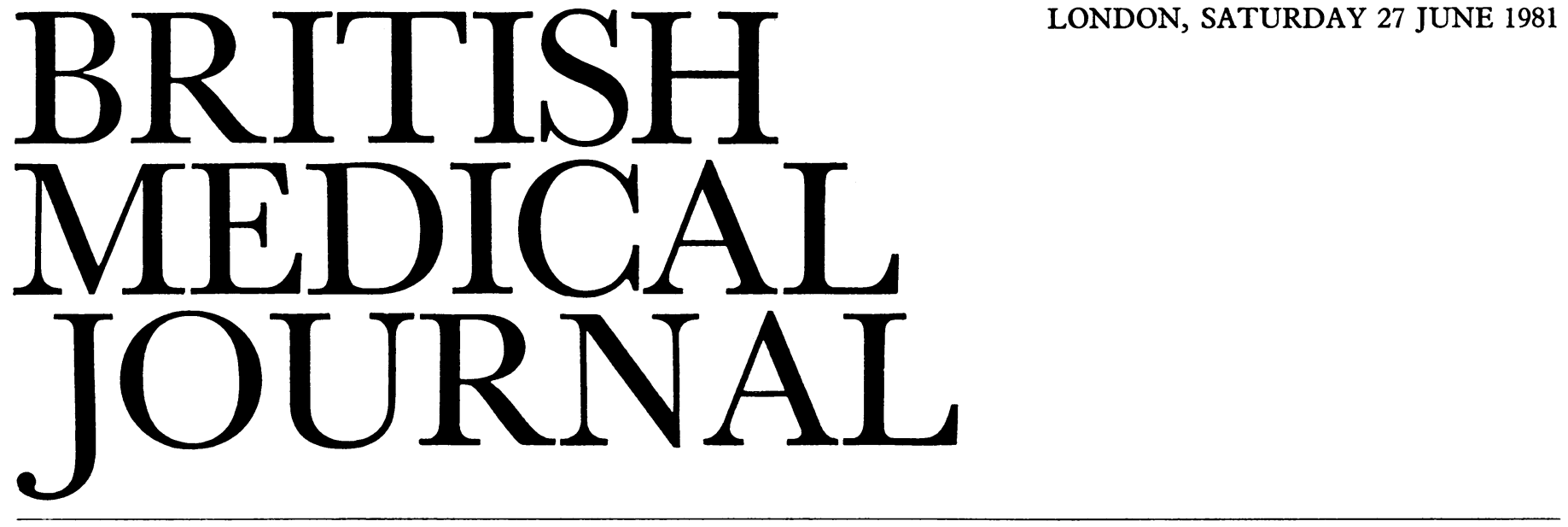

\title{
Breast cancer and the pill-a muted reassurance
}

The evidence implicating endocrinological factors in the cause of breast cancer is good but indirect. Men given oestrogens have an increased risk of developing the disease, men with breast cancer excrete an abnormally high concentration of urinary oestrogens, ${ }^{1}$ and breast cancer is abnormally prevalent in men with Klinefelter's syndrome. ${ }^{2}$

Many of the established risk factors for women developing the disease are probably also related to hormones. An early menarche and late first pregnancy are positive risk factors, while a late menarche and an early artificial menopause are protective. ${ }^{3}$ One current hypothesis suggests that exposure of the breast-duct epithelium to unopposed oestradiol, resulting from an abnormal duration of anovular menstrual cycles at the menarche or just before the menopause, may be the final determinant to which most other established risk factors are related. ${ }^{4}$ If this is correct then we might view with concern the widespread practice of prescribing oestrogen-containing products to otherwise healthy women for prolonged periods of time, especially when the oestrogen is unopposed. Under this cloud of suspicion should fall hormone-replacement treatment for postmenopausal women ${ }^{5}$ and the contraceptive pill for premenopausal women.

So far as the contraceptive pill is concerned two papers in this week's $B M F$ (pp 2089, 2093)offer a cautious reassurance. The first comes from the Royal College of General Practitioners' oral contraceptive study. In this important project a cohort of 23000 women using oral contraceptives was recruited over a 14-month period in 1968 and has been under continuous observation ever since, together with a similar number of matched controls. The relative risks of developing breast cancer for users and non-users have been calculated for the whole group and for various substrata. This study found no convincing evidence that oral contraceptives were linked with breast cancer, though in the age group 30-34 years at the time of entry the risk ratio was $3 \cdot 33$, which just failed to reach statistical significance.

The second study was originally established with the help of the Family Planning Association. Over 17000 women were recruited from family planning clinics in different parts of Britain between the years 1968 and 1974. It reached similar conclusions, with none of the relative risks approaching statistical significance. In particular, it was unable to reproduce the suggestive findings for younger women arising from the Royal College of General Practitioners' study; but (as the authors point out) only 14 women who developed breast cancer were aged under 34 at the time of diagnosis.
Both groups of authors agree that this is not the end of the matter. Concern still remains over the as-yet undetermined effects of duration of exposure to the pill, the possible prolonged latent period between the action of a carcinogen and the appearance of the cancer, and the paucity of information concerning the risk of breast cancer among young women postponing their first pregnancy by using oral contraceptives.

Large-scale use of the pill did not begin until the mid-1960s. The peak age incidence for breast cancer is around 55 years. Young women who began using the pill in, say, 1965 at the age of 20 might not, therefore, be expected to show an increased risk (should one exist) until the year 2000. The Royal College of General Practitioners has emphasised the need for continuing follow-up of the younger women in their study and of those having prolonged exposure to its theoretical hazard.

Neither study has sufficient data to assess the risk to young women using the pill before their first pregnancy. In the Royal College of General Practitioners' study only $15 \%$ of the users were nulliparous at recruitment, while in the Oxford Family Planning Association study the data were derived mostly from women who had started using the pill in their 20 s after giving birth to at least one child.

Some further information on this point has been provided by Pike and his colleagues in a recent paper in the British Fournal of Cancer. ${ }^{6}$ This case-control study of 163 young women with breast cancer aged 32 or less at the time of diagnosis reported a statistically significant trend between the number of months of taking oral contraceptives before the first full-term pregnancy and the risk of developing breast cancer. Young women who had been on the pill for more than 97 months before the birth of their first child had over three times the risk of developing breast cancer before the age of 32 than did matched controls; and the risk appeared to be even greater if there had been a history of benign breast disease. The study by Pike et $a l^{6}$ may be criticised, however, as our first paper ( $p$ 2089) points out: they were able to interview only 163 women from a total of 245 who were eligible, and it is not clear whether they adjusted adequately for differences in their cases and controls in the interval between menarche and first pregnancy.

The findings need, then, to be kept in perspective. By any standards the risk of developing breast cancer under the age of 35 is remote, and undoubtedly many women would accept over three times this small risk for the benefits and confidence offered by oral contraceptives. Nevertheless, the implications are worrying: if this risk persists into middle age-a possibility 
that cannot be proved or disproved by any of the studies so far cited-it might eventually produce groups of women with perhaps a one-in-five chance of developing breast cancer in their lifetimes.

Attempting to offer recommendations on the basis of these data is an awesome responsibility. An excessively alarmist response could result in an epidemic of unplanned pregnancies, with all the attendant social and medical consequences they entail; yet to provide blanket reassurance might prove to be irresponsible. The "worst-case" conclusion is that young girls should avoid prolonged use of the pill before their first pregnancy, especially if they have a history of benign breast disease. Alternatively, girls starting the pill in their teens might be included among the "at-risk" population offered screening, which on present evidence can be recommended only for women over the age of $50 .^{8}$

${ }^{1}$ Dao TL, Morreal C, Nemoto T. Urinary estrogen excretion in men with breast cancer. N Engl f Med 1973;289:138-40.

${ }^{2}$ Dodge OG, Jackson AW, Muldal S. Breast cancer and interstitial-cell tumor in a patient with Klinefelter's syndrome. Cancer $1969 ; 24: 1027-32$.

${ }^{3}$ Macmahon B, Cole P, Brown J. Etiology of human breast cancer : a review. Fournal of the National Cancer Institute 1973;50:21-42.

${ }^{4}$ Short RV, Drife JO. The aetiology of mammary cancer in man and animals. Symposia-Zoological Society of London 1977;41:211-30.

${ }^{5}$ Hoover R, Gray LA, Cole P, Macmahon B. Menopausal estrogens and breast cancer. $N$ Engl f Med 1976;295:401-5.

${ }^{6}$ Pike MC, Henderson BE, Casagrande JT, Rosario I, Gray GE. Oral contraceptive use and early abortion as risk factors for breast cancer in young women. Br f Cancer $1981 ; 43: 72-6$.

${ }^{7}$ Donegan WL. Epidemiology. In: Donegan WL, Spratt JS, eds. Cancer of the breast. Philadelphia: W B Saunders Co, 1979:18-20.

${ }^{8}$ Bailar JC. Screening for early breast cancer: pros and cons. Cancer 1977; $39: 2783-95$.

\section{Non-rheumatic mitral regurgitation}

The mitral valve has a complex functional anatomy. Its competence depends on the left atrial wall, mitral annulus, valve leaflets, chordae tendineae, papillary muscles, and left ventricle. ${ }^{1}$ Mitral regurgitation may result from disease of any of these structures, but moderate to severe regurgitation usually results from disease of the valve leaflets or subvalvar apparatus, classically caused by chronic rheumatic heart disease. Yet now that acute rheumatic fever has become rare in Britain new cases of mitral regurgitation continue to present.

Non-rheumatic mitral regurgitation is a useful term, which may include causes such as infective endocarditis, myocardial infarction with papillary muscle necrosis and avulsion of the chordae, and the "floppy" mitral valve. This last category is now recognised as being a common cause of isolated mitral regurgitation, and may be differentiated from rheumatic disease not just by the pathological appearances but also by echocardiography. The rheumatic valve is thickened and relatively immobile, whereas the floppy valve is thin, abnormally mobile, and may prolapse into the left atrium during systole. The floppy valve may be associated with rupture of the chordae tendineae, which will precipitate sudden severe mitral regurgitation and pulmonary oedema.

Acute mitral regurgitation carries a poor prognosis, and surgical treatment should be recommended. The survival of patients with chronic mitral regurgitation is better and nearly
$60 \%$ are alive 10 years later. ${ }^{2}$ Some patients, however, will become disabled by exertional dyspnoea and palpitation, and in this group again surgical treatment should be considered. The results of cardiac surgery are largely determined by the state of the heart muscle, which will gradually deteriorate if subjected to chronic overloading, as in mitral regurgitation. When the myocardium remains healthy but while the natient has few symptoms, early operation gives good results; when the patient has severe symptoms with irreversible left ventricular dilatation and fibrosis, surgery gives poor results. Somehow a compromise must be achieved so that surgery may be recommended before the left ventricle becomes too badly damaged.

Left ventricular performance can be assessed by measurements of pressure, volume, and flow. Measures of left ventricular volume may be derived from angiography, and the ejection fraction (the stroke volume divided by the enddiastolic volume) has proved most helpful in predicting the outcome of surgical treatment. ${ }^{3}$ Usually a low ejection fraction indicates poor left ventricular function and a smaller chance of recovery after surgery. In mitral regurgitation, however, the left ventricular blood can leak back into the left atrium and this low pressure outlet allows the ejection fraction to remain normal even when the myocardium is severely damaged. Two recent papers have suggested that the left ventricular end-systolic ${ }^{4}$ or diastolic volume ${ }^{5}$ may be a better predictor of the ventricular performance after valve replacement in patients with mitral regurgitation-though a low ejection fraction is uniformly associated with a poor outcome. A simple predictor of a good result is a history of symptoms that is less than one year.

Left ventricular angiography requires left heart catheterisation (though this may change with the development of nuclear angiography). In contrast, echocardiography allows one dimension of the left ventricle to be studied repeatedly without harm or discomfort to the patient. Left ventricular end-systolic and diastolic shortening of the echocardiographic dimension reflects the ejection fraction. In patients with mitral regurgitation who remain well all these indices of left ventricular performance are increased, but as the left ventricle fails the fractional shortening falls to normal again. ${ }^{6}$ Thus the echocardiogram can both help the cardiologist make the diagnosis of non-rheumatic mitral regurgitation and give him an indication of left ventricular performance which may serve as a guide to the timing of surgery. Schuler and his colleagues ${ }^{8}$ have shown that patients with end-systolic and diastolic dimensions of greater than 5.0 and $7.0 \mathrm{~cm}$ respectively, coupled with a low normal or depressed ejection fraction, continue to have impaired left ventricular function after surgery, with the implication that these patients have a poor prognosis.

The other important factor that influences the results of surgery in patients with mitral regurgitation is the nature of the operation. The alternatives are repairing the valve or replacing it with a prosthesis or biological valve. Reconstructive surgery has its advocates, ${ }^{9}$ but most British surgeons prefer to replace the valve. Prosthetic valves, though imperfect, are well tried, and the Björk valve may be preferable to the Starr. ${ }^{5}$ They require long-term anticoagulant treatment and if this is contraindicated then a porcine heterograft may be the better option. $^{10}$

\footnotetext{
' Perloff JK, Roberts WC. The mitral apparatus. Functional anatomy of mitral regurgitation. Circulation $1972 ; 46: 227-39$.

2 Rapaport E. Natural history of aortic and mitral valve disease. Am f Cardiol
} $1975 ; 35: 221-7$. 